\title{
Expression of $b c l-2$ and Epstein-Barr virus LMP1 in lymphocytic interstitial pneumonia
}

\author{
Philomena M Kaan, Richard G Hegele, Shizu Hayashi, James C Hogg
}

\begin{abstract}
Background - Epstein-Barr virus (EBV) genome has been demonstrated in lung tissues of patients with lymphocytic interstitial pneumonia (LIP) but its role in the pathogenesis of this condition is unclear. In vitro studies have shown that EBV can immortalise and transform cells by upregulation of the cellular proto-oncogene, B cell leukaemia-2 (bcl-2), via the viral latent membrane protein, LMP1. The purpose of this study was to determine whether bcl-2 expression is upregulated in the lungs of patients with LIP and whether EBV LMP1 has a role in this bcl-2 expression.

Methods - Immunohistochemical analysis using alkaline phosphatase antialkaline phosphatase (APAAP) was performed on formalin fixed, paraffin embedded lung tissues from 13 patients with LIP using anti-LMP1 and anti-bcl-2 monoclonal antibodies. Lung tissues from nine patients with idiopathic pulmonary fibrosis (IPF) and nine necropsy cases without pulmonary disease served as controls. LMP1 positivity was estimated as the number of LMP1 positive cells per unit area of lung tissue. Immunostaining for bcl-2 expression was assessed by a pictorial-based semiquantitative grading system.
\end{abstract}

Results - Positive immunostaining for LMP1 was localised to airway epithelial cells of lung tissue. Ten out of $13(77 \%)$ patients with LIP were positive for LMP1 compared with three of nine cases $(33 \%)$ in each control group. LMP1 positivity of LIP cases was significantly greater than that of non-LIP cases: LIP versus IPF (mean difference, $\mathbf{9 5 \%}$ confidence interval (CI)) 2.39 (1.54 to 3.24); LIP versus necropsy controls 2.62 (1.77 to 3.47$)$. bcl-2 immunostaining was localised to lymphocytes within the alveolar septa and lymphoid aggregates of patients with LIP. The cumulative score for $b c l-2$ immunostaining was significantly higher in the lungs of patients with LIP than in those of patients with IPF and necropsy controls: LIP versus IPF and LIP versus necropsy controls (mean difference, 95\% CI) 7.55 (7.18 to 7.92$)$.
Conclusions - These immunohistochemical studies have shown the presence of EBV LMP1 protein in airway epithelial cells and overexpression of the cellular bcl2 protein in lymphoid cells of lung tissue in patients with LIP. These geographically distinct staining patterns of immunostaining suggest that the involvement of EBV LMP1 in the upregulation of cellular bcl-2 is more complex in LIP than was thought from previous in vitro observations. The respective roles of $\mathrm{EBV}$ LMP1 and $b c l-2$ in the pathogenesis of LIP require further studies.

(Thorax 1997;52:12-16)

Keywords: Epstein-Barr virus, lymphocytic interstitial pneumonia, B cell leukaemia-2 gene.

Lymphocytic interstitial pneumonia (LIP) is a pulmonary lymphoproliferative disorder of unknown aetiology that is characterised by expansion of the alveolar septa by prominent mononuclear cell infiltrates. ${ }^{1}$ Several lines of evidence have implicated the Epstein-Barr virus (EBV) in the aetiology and pathogenesis of LIP. Andiman et al demonstrated the EBV genome by Southern blot hybridisation in eight out of 10 lung biopsy specimens showing LIP. Barbera et $a l^{3}$ used in situ hybridisation to demonstrate the internal repeat sequence of the EBV genome in lung biopsy specimens from nine of 14 adult patients with LIP compared with only two of 10 patients with idiopathic pulmonary fibrosis (IPF) who served as controls. In addition, significantly higher titres of EBV antibodies have been measured in patients with LIP who have AIDS than in HIV positive patients without LIP. ${ }^{45}$ These reports have established that the EBV genome resides in the lungs of patients with LIP and that the virus is capable of eliciting a specific host immune response, but the role of EBV in the pathogenesis of LIP remains unclear.

Aberrant expression of the B cell leukaemia2 (bcl-2) proto-oncogene is involved in the pathogenesis of a wide variety of lymphoproliferative disorders. ${ }^{67}$ Several categories of oncogenes are based on the effects of protein products of these genes on normal tissue homeostasis. Growth proliferating genes such as the nuclear transcription factor $m y c$, and 
tumour suppressor genes such as the p53 gene destroy the normal tissue homeostasis by either promoting or inhibiting cell growth and proliferation. In contrast, $b c l-2$ represents a distinct category of oncogenes by its effects on apoptosis (programmed cell death). ${ }^{11}$ It normally plays an important role in regulating apoptosis during $\mathrm{B}$ cell development ${ }^{8}$ and in the maintenance of B cell memory. ${ }^{9}{ }^{10}$ However, overexpression of $b c l-2$ has been associated with prolonged cell survival, which favours the acquisition of secondary genetic events that could result in neoplasia ${ }^{11}$ and production of low grade lymphoproliferative disorders such as LIP.

We have examined the expression of the EBV latent membrane protein (LMP1) and that of the human $b c l-2$ oncoprotein because LMP1 has both an essential role in cell transformation ${ }^{12}$ and can induce upregulation of $b c l$ 2 in vitro. ${ }^{1314}$ Our objective was to determine whether the lungs of patients with LIP show upregulation of $b c l-2$ expression compared with the lungs of patients with the usual form of interstitial pneumonia (IPF) and normal lung tissue from patients who died without lung disease.

\section{Methods}

PATIENT MATERIAL

Formalin fixed, paraffin embedded lung tissues were analysed from 13 patients with LIP collected from several sources, nine patients with IPF and from nine necropsy cases where death occurred from non-respiratory causes. The patients with LIP (mean (SD) age 56.1 (9.0) years) and those with IPF (mean (SD) age 59.7 (7.2) years) have been reported as part of an earlier study. ${ }^{3}$ There were no serological data for EBV available on any of these patients. In the LIP group five patients had concomitant conditions that included AIDS $(n=2)$, Sjogren's syndrome $(n=2)$, and hypergammaglobulinaemia $(n=1)$. The necropsy cases with normal lungs consisted of seven men and two women of mean (SD) age 64.0 (15.7) years and were obtained from the necropsy service of the department of pathology at St Paul's Hospital. The causes of death in these cases included cardiac related illnesses $(n=7)$, intracerebral haemorrhage $(n=1)$, and trauma $(n=$ 1).

Formalin fixed, paraffin embedded tissue from a case of nasopharyngeal carcinoma and lymph node tissue from a case of follicular lymphoma served as positive tissue controls for anti-LMP1 and anti-bcl-2 monoclonal antibodies, respectively. In addition, Namalwa cells, a lymphoblastoid cell line containing two copies of EBV per cell, ${ }^{15}$ were included as a positive control for determining the sensitivity of LMP1 immunostaining.

IMMUNOHISTOCHEMICAL ANALYSIS

Mouse monoclonal antibody against the EBV LMP1 (CS1-4) and human bcl-2 were purchased from Dakopatts (Glostrup, Denmark). All specimens were cut into $5 \mu \mathrm{m}$ sections using a fresh microtome blade for each block to avoid any possible contamination from carry-over. Four sections from each block were used for staining with the two monoclonal antibodies and with appropriate concentrations of non-

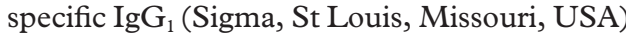
as negative controls. After dewaxing in xylene the sections were transferred to silane coated slides and treated by boiling in $10 \mathrm{mM}$ citric acid ( $\mathrm{pH}$ 6-6.3) to unmask antigenic sites. ${ }^{16}$ The standard protocol for the alkaline phosphatase anti-alkaline phosphatase (APAAP) technique ${ }^{17}$ was employed with the rabbit antimouse immunoglobulin (Dakopatts, 1 in 20 dilution) serving as secondary antibody. The anti-LMP1 monoclonal antibody was diluted 1 in 50 as suggested by the suppliers while the anti-bcl-2 monoclonal antibody was diluted 1 in 400 as this concentration of the anti-bcl-2 monoclonal antibody facilitated detection of cells exhibiting high level expression of the $b c l$ 2 gene. All immunohistochemical staining was carried out in parallel with staining by nonspecific mouse $\operatorname{IgG}_{1}$ at a concentration comparable to that of the test antibody. The specificities of both the monoclonal antibodies were assessed by comparison of the test section with the corresponding control section stained with mouse $\mathrm{IgG}_{1}$. After completion of immunostaining the sections were counterstained with haematoxylin. The slides were coded prior to microscopic examination which was carried out without knowledge of the tissue source. The sensitivity of the anti-LMP1 monoclonal antibody was assessed by determining the percentage of positively stained cells in sections prepared from formalin fixed, paraffin embedded blocks of Namalwa cells.

ESTIMATION OF LMP1 POSITIVE CELLS PER UNIT AREA

The total number of cells (n) stained by the anti-LMP1 monoclonal antibody in each lung section was counted using a hand held counter. The cross sectional area of each lung section was determined using the Bioquant BQ System IV software (R\&M Biometrics Inc, Nashville, Tennessee, USA). This software program contains a feature which integrates a measured circumferential distance to calculate the enclosed area. The cross sectional area (A) of the lung tissue section was obtained as the mean of the integrated areas from three measurements of tissue outline. To correct for variation in cellularity in the different groups of specimens the volume fraction of tissue component (f) was estimated by the method of point counting. ${ }^{18}$ The LMP1 positivity, which is defined as the number of cells expressing LMP1 per unit area of lung tissue, was calculated according to the formula:

$$
\mathrm{LMP} 1 \text { positivity }=\frac{\mathrm{n}}{\mathrm{f} \times \mathrm{A}}
$$

where $\mathrm{n}=$ the number of LMP1 positive cells, $\mathrm{f}=$ volume fraction of lung corresponding to tissue, and $\mathrm{A}=$ cross sectional area of the lung tissue. 


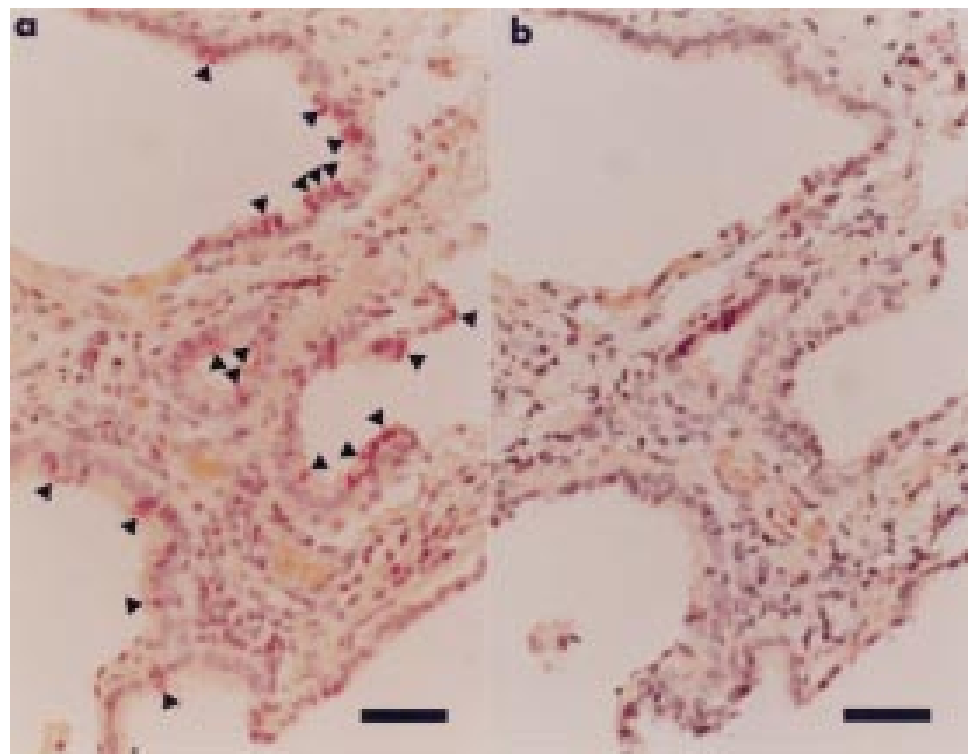

Figure 1 Photomicrographs of LMP1 immunostaining of lung tissue from a patient with lymphocytic interstitial pneumonia (LIP): (a) LMP1 protein localised to the apical region of epithelial cells (arrow heads); (b) IgG negative control. APAAP reaction; bar represents $50 \mu \mathrm{m}$.

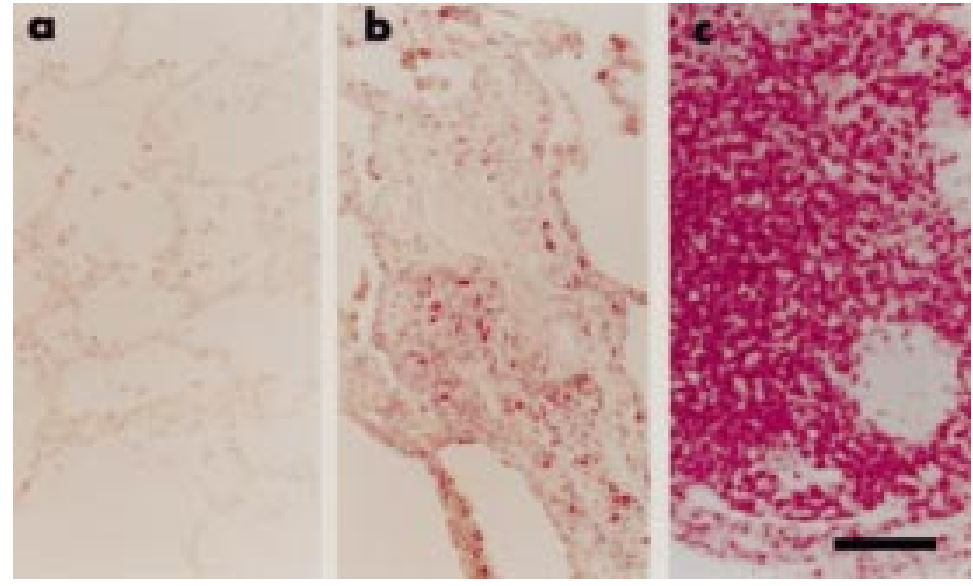

Figure 2 bcl-2 immunostaining of representative lung sections from (a) a necropsy control, (b) a patient with idiopathic pulmonary fibrosis (IPF), and (c) a patient with lymphocytic interstitial pneumonia (LIP). Positive bcl-2 immunostaining is observed in lymphocytes within the interstitial space (b) and in cells comprising lymphoid aggregates in LIP (c). APAAP reaction; bar represents $50 \mu \mathrm{m}$.

GRADING SYSTEM FOR BCL-2 EXPRESSION

$b c l-2$ expression was assessed based on a histological semiquantitative grading system of $b c l-$ 2 immunostaining ranging from 0 (none) to 3 (high). Ten randomly selected fields from each slide were evaluated and the sum of scores represented the cumulative score for that slide. To assess the reproducibility of the scoring system the intra-observer and inter-observer variations were determined and expressed as the coefficients of variation, $\mathrm{R}_{2} / \mathrm{R}_{2 \max } \cdot{ }^{19} \mathrm{R}_{2} / \mathrm{R}_{2 \max }$ values of $\geq 0.75$ were considered to be acceptable. ${ }^{19}$ The coefficient of intra-observer variation was established by the same observer (PMK) scoring 100 randomly selected fields two months after the first scoring. The interobserver variation was determined by two individuals (PMK and RGH) scoring 100 ran-
Table 1 Prevalence of EBV LMP1 in lung tissues

\begin{tabular}{llcl}
\hline & LIP & IPF & Necropsy \\
\hline No. of positive cases & $10 / 13^{*}$ & $3 / 9$ & $3 / 9$ \\
& $(77 \%)$ & $(33.3 \%)$ & $(33.3 \%)$ \\
No. of positive blocks & $20 / 26^{*}$ & $5 / 20$ & $3 / 18$ \\
& $(77 \%)$ & $(25 \%)$ & $(17 \%)$ \\
\hline
\end{tabular}

$\mathrm{LIP}=$ lymphocytic interstitial pneumonia $\mathrm{IPF}=$ idiopathic pul monary fibrosis.

$* \mathrm{p} \leq 0.05$ LIP versus IPF and LIP versus necropsy controls.

domly selected fields simultaneously using a teaching microscope.

\section{STATISTICAL ANALYSIS}

The prevalence of EBV among the three groups was analysed using contingency table analysis ( $\chi^{2}$ statistics) with Tukey-type testing to correct for multiple comparisons. ${ }^{20}$ Of the cases that were LMP1 positive, the data were transformed by logarithmic operation, analysed by the method of single factor analysis of variance (ANOVA), and corrected for multiple comparisons using the sequential rejective Bonferroni procedure. ${ }^{21}$ The mean cumulative scores for $b c l-2$ immunostaining were analysed using ANOVA. All calculations were done using Systat Version 6 (Evanston, Illinois, USA). The results were reported as the least squares estimates for the mean differences (with 95\% CI) of LMP1 positivity and $b c l-2$ immunostaining between the groups.

\section{Results}

IMMUNOHISTOCHEMISTRY: ANTI-LMP1

MONOCLONAL ANTIBODY

LMP1 positive cells in the lung tissues from all three patient groups were of epithelial origin and were found primarily along bronchioles (fig 1a). Diffuse LMP1 signals were localised in the apical region of these epithelial cells and were not observed in lymphoid cells in any of the patient samples. No immunostaining was observed in sections incubated with nonspecific $\operatorname{IgG}_{1}$ antibody (fig $1 \mathrm{~b}$ ).

The prevalence of EBV LMP1 in lung tissues is summarised in table 1. Positive LMP1 immunostaining was observed in 10 out of 13 $(77 \%)$ patients with LIP and three of nine $(33 \%)$ cases in both control groups $(\mathrm{p} \leq 0.05)$. LMP1 positivity (mean difference, $95 \% \mathrm{CI}$ ) of LIP cases was significantly greater than that of non-LIP cases: LIP versus IPF 2.39 (1.54 to 3.24); LIP versus necropsy controls 2.62 (1.77 to 3.47). There was no significant difference in LMP1 positivity between the patients with IPF and the necropsy control group. In $5 \mu \mathrm{m}$ sections prepared from formalin fixed, paraffin embedded Namalwa cells $78 \%$ of cells showed positive immunostaining for LMP1 (data not presented).

IMMUNOHISTOCHEMISTRY: HUMAN ANTI-BCL-2 MONOCLONAL ANTIBODY

In the lung tissue sections examined positive immunostaining for $b c l-2$ protein was restricted to cells of lymphoid origin (fig 2). Figure 3 


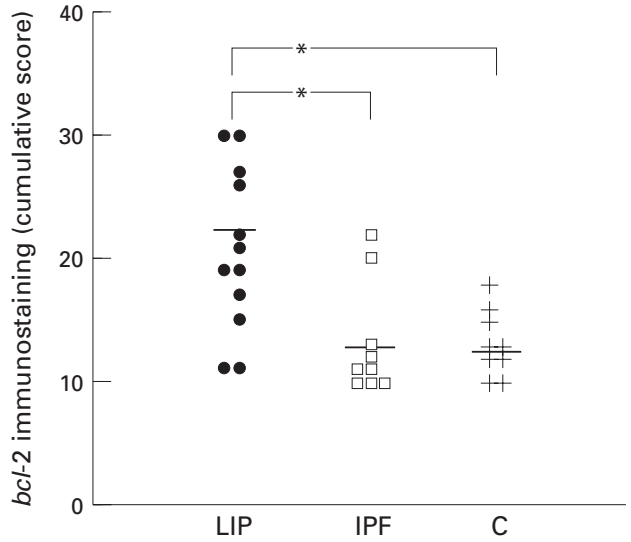

Figure 3 Semiquantitative scoring of bcl-2 immunostaining of lung sections from patients with lymphocytic interstitial pneumonia (LIP) $(\mathbf{O}, n=13)$, idiopathic pulmonary fibrosis (IPF) $(\square, n=9)$ and necropsy controls $(+, n=9)$. Each symbol represents the cumulative score from 10 randomly selected fields examined for each individual case. The mean of the cumulative scores of each group is indicated by a horizontal bar. ${ }^{*} p<0.005$.

shows the distribution of the cumulative scores for $b c l-2$ immunostaining in the lung sections from the three groups of patients. The cumulative score for $b c l-2$ immunostaining (mean difference, $95 \%$ CI) was significantly higher in the LIP group than in those with IPF or the necropsy control groups: 7.55 (7.18 to 7.92 ). There was no significant difference in the mean cumulative scores between the IPF and necropsy control groups.

The reproducibility of the $b c l-2$ scoring system was validated through the analysis of intraobserver and inter-observer coefficients of variation. The $R_{2} / R_{2 \max }$ value of intra-observer variation on $b c l-2$ assessment was 0.97 and the inter-observer $R_{2} / R_{2 \max }$ value was 0.84 .

\section{Discussion}

The results of this study show a greater prevalence and density of EBV LMP1 and higher levels of $b c l-2$ expression in lung tissue from patients with LIP than in those with IPF and non-diseased lungs. Previous studies have demonstrated evidence of EBV genome in the lung of patients with $\mathrm{LIP}^{23}$ and a virus-specific host immune response. ${ }^{45}$ In addition, bronchoalveolar lavage studies ${ }^{22}$ have suggested that the site of viral replication in the epithelial cells of the oropharynx could be extended to the peripheral lung, and that the lung may serve as a reservoir for EBV replication. This concept was further supported by the observations of Egan $e^{2} \mathrm{al}^{23}$ who reported a high prevalence of the EBV viral capsid antigen (VCA) and gp $340 / 220$ glycoprotein in the lungs of patients with IPF. The lower prevalence of EBV protein in the lungs of patients with IPF in the current study may be related to differences in populations being examined and/or technical factors - for example, lung tissue processing, preservation of epitopes in lung tissue, differences in target epitopes (VCA and gp340/220 versus LMP1) - of immunohistochemical analysis. In any event the presence of LMP1 positive cells in the IPF and necropsy control groups in the current study is consistent with the serological evidence of EBV infection in the general population, ${ }^{24}$ and the greater EBV density documented in lung tissue from LIP patients compared with the two control groups suggests that a more active EBV infection was present in LIP.

High levels of $b c l-2$ expression have been reported in a wide variety of lymphoproliferative disorders ${ }^{67}$ and our study extends these reports by establishing that there is a high level of $b c l-2$ expression in LIP. Despite conflicting reports of whether the lymphocytic subpopulation in LIP is primarily of $\mathrm{B}$ or $\mathrm{T}$ cell lineage, we have shown in a previous study ${ }^{3}$ that $\mathrm{B}$ cells were the main constituent of the pulmonary lymphoid aggregates in these LIP patients. Thus, the distribution of $b c l-2$ immunostaining observed in this study was within B cell-rich areas. Increased expression of $b c l-2$ confers a survival advantage on B cells by protecting against apoptosis. This extended cell survival could contribute to the increased number of lymphocytes observed in the tissue in LIP, and the increased opportunity for acquiring secondary defects in either growth factor or tumour suppressor genes ${ }^{11}$ might account for the frequent progression of LIP to low or mid grade non-Hodgkin's lymphoma.

Studies of EBV infected lymphoblastoid cell lines have suggested that LMP1 is directly involved in upregulation of $b c l-2$ expression ${ }^{1325}$ in vitro, whereas the current study revealed LMP1 and $b c l-2$ immunostaining within different cell types in lung tissue from patients with LIP. Replicative EBV infection with shedding of the virus from the cell surface usually occurs in epithelial cells and involves the expression of all of the approximately 80 genes encoded by the virus. Infection and transformation of lymphocytes, on the other hand, results from organisation of the viral genome into a circular extrachromosomal episome that resides in the cell nucleus with expression of only about 10 viral genes ${ }^{26}$ including LMP1. In contrast to other investigators (reviewed by Niedobitek et $a l^{27}$ ) who have suggested that the lymphoid system is the site of EBV persistence in vivo, our observations clearly show evidence of viral protein within lung epithelial cells. The expression of LMP1 in epithelial cells could therefore be serving as a marker for EBV infection of the lung, rather than as the direct cause of the increased $b c l-2$ expression by the infiltrating lymphocytes. Alternatively, the paucity of LMP1 signals in lymphoid cells in lung tissue of patients with LIP could be explained by the differences between cellular interactions in vitro and in vivo. ${ }^{2829}$ For example, LMP1 expression can be demonstrated in cultured Namalwa cells that are derived from Burkitt's lymphoma, whereas Western blotting of protein extracted from Burkitt's lymphoma cells obtained by biopsy does not reveal LMP1 protein. ${ }^{28}$ Other groups have also observed no direct correlation between LMP1 and $b c l-2$ expression in other lymphoproliferative disorders. $^{3031}$ The absence of LMP1 staining 
of lymphocytes in LIP cannot be attributed to a lack of sensitivity for the anti-LMP1 monoclonal antibody because a high percentage $(78 \%)$ of Namalwa cells, fixed in formalin and embedded in paraffin in a similar manner to the lung tissue specimens, showed positive LMP1 immunostaining.

In summary, we have found a higher prevalence and density of EBV LMP1 expression in bronchiolar epithelial cells in LIP associated with increased $b c l-2$ immunostaining in lymphoid cells. These results suggest that EBV infection has a role in the pathogenesis of LIP, but that the geographically distinct patterns of LMP 1 and $b c l-2$ immunostaining indicate that the specific viral-cellular interactions which result in overexpression of $b c l-2$ remain to be determined.

The authors are indebted to the following clinicians who provided pathological specimens and patient information: Dr TV Colby (Scottsdale, AZ); Dr HE Manson (Richmond, BC); Dr DA Owen (Vancouver, BC); Dr G Montessori (New Westminister, BC); Dr F Murphy (Saskatoon, SK), and Dr DJ Mulholland (Kelowna, BC). We thank Mr S Greene for photographic illustration and Dr Barry Wiggs and Ms L Verburgt for statistical consultations.

This work was supported by the Respiratory Health Network of Centers of Excellence. Drs Hegele and Hayashi are recipients of a British Columbia Lung Association/Medical Research of the Roberta Bondar Scholarship. The project was supported by grants from the Medical Research Council of Canada ( 4219 and 12713) and the National Institute of Health (5-56617).

1 Carrington CB, Liebow AA. Lymphocytic interstitial pneumonia. Am $\mathcal{7}$ Pathol 1966;48:36.

2 Andiman WA, Martin K, Rubinstein A, et al. Opportunistic lymphoproliferations associated with Epstein-Barr viral DNA in infants and children with AIDS. Lancet 1985;ii: 1390-3

3 Barbera JA, Hayashi S, Hegele RG, Hogg JC. Detection of Epstein-Barr virus in lymphocytic interstitial pneumonia by in situ hybridization. Am Rev Respir Dis 1992;145: 940-6.

4 Katz BZ, Berkman AB, Shapiro ED. Serologic evidence of active Epstein-Barr virus infection in Epstein-Barr virusassociated lymphoproliferative disorders of children with acquired immunodeficiency syndrome. I Pediatr 1992; 120:228-32

5 Kramer MJ, Saldana MJ, Ramos M, Pitchenik AE. High titers of Epstein-Barr virus antibodies in adult patients with lymphocytic interstitial pneumonitis associated with with lymphocytic interstitial pneun.

6 Zutter M, Hockenbery D, Silverman GA, Korsmeyer SJ. Immunolocalization of the $b c l-2$ protein within hematopoietic neoplasms. Blood 1991; 78:1062-8.

7 Mariano MT, Moretti L, Donelli A, et al. Bcl-2 gene expression in hematopoietic cell differentiation. Blood 1992; 80:768-75.

8 Merino R, Ding L, Veis DJ, Korsmeyer SJ, Nunez G. Developmental regulation of the $b c l-2$ protein and susceptibility to cell death in B lymphocyte. EMBO F 1994; 13:683-91.

9 Nunez G, Hockenbery D, McDonnell J, Sorensen CM, 1991;353:71-3.
10 Liu YJ, Mason DY, Johnson GD, et al. Germinal center cells express $b c l-2$ protein after activation by signals which prevent their entry into apoptosis. Eur f Immunol 1991 21:1905-10.

11 Korsmeyer SJ. $\mathrm{Bcl}-2$ initiates a new category of oncogenes: regulators of cell death. Blood 1992;80:879-86.

12 Wang F, Gregory CD, Sample C, et al. Epstein-Barr virus latent membrane protein (LMP1) and nuclear proteins 2 and $3 \mathrm{C}$ are effectors of phenotypic changes in $\mathrm{B}$ lymphocytes: EBNA-2 and LMP1 cooperatively induce CD23. I Virol 1990;64:2309-18

13 Gregory CD, Dive C, Henderson S, Smith CA, Williams GT, Gordon J. Activation of Epstein-Barr virus latent genes protects human B cells from death by apoptosis. Nature 1991;349:612-4.

14 Finke J, Fritzen R, Ternes P, et al. Expression of $b c l-2$ in Burkitt's lymphoma cell lines: induction by latent membrane Epstein-Barr virus genes. Blood 1992;80:459-69.

15 Lawrence JB, Villnave CA, Singer RH. Sensitive, highresolution chromatin and chromosome mapping in situ: presence and orientation of two closely integrated copies presence and orientation of two closely integrated
of EBV in a lymphoma line. Cell 1988;52:51-61.

16 Cattoretti G, Stefano P, Parravicini C, et al. Antigen unmasking on formalin-fixed, paraffin-embedded tissue sections. F Pathol 1993;171:83-98.

17 Cordell JL, Falini B, Erber WN, et al. Immunoenzymatic labeling of monoclonal antibodies using immune complexes of alkaline phosphatase and monoclonal antialkaline phosphatase (APAAP complex). I Histochem Cytochem 1984;32:219-29.

18 Bolender RP, Hyde DM, Dehoff RT. Lung morphometry: a new generation of tools and experiments for organ, tissue, cell, and molecular biology. Am 7 Physiol (Lung Cell Mol Physiol) 1993;265:L521-48.

19 Conover WJ. Measures of dependence. In: Practical nonparametric statistics. New York: McGraw-Hill, 1988.

$20 \mathrm{Zar} \mathrm{JH}$. The binomial distribution. In: Biostatistical analysis. New Jersey: Prentice-Hall, 1984:400-2.

21 Holland BS, Copenhaver MD. An improved sequentially rejective Bonferroni test procedure. Biometrics $1987 ; \mathbf{4 3}$ : $417-23$.

22 Lung ML, Lam WK, So SY, Lam WP, Chan KH, Ng $\mathrm{MH}$. Evidence that respiratory tract is major reservoir for Epstein-Barr virus. Lancet 1985;i:889-92.

23 Egan JJ, Stewart JP, Hasledton PS, Arrand JR, Carroll $\mathrm{KB}$, Woodcock AA. Epstein-Barr virus replication within pulmonary epithelial cells in cryptogenic fibrosing alveolitis. Thorax 1995;50:1234-9.

24 Rocchi G, DeFelici A, Ragona G, Heinz A. Quantitative evaluation of Epstein-Barr virus-infected mononuclea peripheral blood leukocytes in infectious mononucleosis. N Engl f Med 1977;296:132.

25 Rowe M, Peng-Pilon M, Huen DS, et al. Upregulation of $b c l-2$ by the Epstein-Barr virus latent membrane protein LMP1: a B-cell specific response that is delayed relative to NF-KB activation and to induction of cell surface markers. I Virol 1994;68:5602-12.

26 Straus SE, Cohen JI, Tosato G, Meier J. Epstein-Barr virus infections: biology, pathogenesis, and management. Ann Intern Med 1993;118:45-58.

27 Niedobitek G, Young LS. Epstein-Barr visu persistence and virus-associated tumors. Lancet 1994;343:333-5.

28 Rowe M, Rowe DT, Gregory CD, et al. Differences in B cell growth phenotype reflect novel patterns of Epsteincell growth phenotype reflect novel patterns of EpsteinBarr virus latent gene expression
cells. EMBO $71987 ; 6: 2743-51$.

29 Gregory CD, Rowe M, Rickinson AB. Different EpsteinBarr virus-B cell interactions in phenotypically distinct clones of a Burkitt's lymphoma cell line. F Gen Virol 1990 71:1481-95.

30 Tao Q, Srivastava G, Loke SL, Ho FCS. Lack of correlation between expression of Epstein-Barr virus (EBV) laten membrane protein and $b c l-2$ oncoprotein in vivo. 7 Clin Pathol 1994;47:589-91.

31 Schlaifer D, Brousset P, Attal M, et al. bcl-2 proto-oncogene and Epstein-Barr virus latent membrane protein-1 expression in AIDS-related lymphoma. Histopathology 1994 25:77-82. 